\title{
Indonésie: Des études coordonnées sont nécessaires á l'évaluation des tendances
}

Frontiers in Reproductive Health

Follow this and additional works at: https://knowledgecommons.popcouncil.org/departments_sbsr-rh

Part of the International Public Health Commons, Maternal and Child Health Commons, and the Work, Economy and Organizations Commons

How does access to this work benefit you? Let us know!

\section{Recommended Citation}

"Indonésie: Des études coordonnées sont nécessaires á l'évaluation des tendances," FRONTIERES

Résumés de Recherche Opérationnelle. Dakar: Population Council, 2000.

This Brief is brought to you for free and open access by the Population Council. 


\title{
Indonésie Institutionnalisation de la $R O$ \\ Des études coordonnées sont nécessaires à l'évaluation des tendances
}

$N^{\circ} 8$

\begin{abstract}
Des études longitudinales avec des indicateurs pertinents et une population représentative sont indispensables à la détermination des changements apportés aux indicateurs de santé maternelle et infantile.
\end{abstract}

\section{Contexte}

En 1999, le Population Council/Indonésie a entrepris une revue critique de 11 enquêtes et études indonésiennes évaluant les divers indicateurs de santé maternelle et infantile (SMI) entre 1996 et 1999. Plusieurs de ces études ont tenté d'établir un lien entre ces indicateurs et la crise économique que traversait le pays depuis juillet 1997. Les agents du Council se sont efforcés à expliquer comment ces études étaient parvenues à des conclusions divergentes.

\section{Résultats}

- La programmation des activités de collecte des données et leur couverture géographique sont les facteurs déterminants qui ont induit les différences entre les résultats.

- Les données doivent être désagrégées au niveau le plus bas possible, afin de vérifier les différences d'impact entre les régions, entre les groupes d'âge et les groupes socioéconomiques, les différences selon le genre, et selon la résidence.
- L'explication, par la crise économique, des changements survenus au niveau des indicateurs de SMI peut se révéler trompeuse. L'état nutritionnel et sanitaire semble avoir commencé à se dégrader avant que ne débute la crise. L'impact général de la crise ne s'est peut être pas fait sentir sur la SMI pendant plusieurs années.

- Les services de planification familiale ont connu certaines perturbations entre 1997 et 1999. Le prix des contraceptifs s'est accru vers la fin de 1997 et le début de 1998, et il a beaucoup varié entre mi-1998 et mi-1999. Les cliniques ont enregistré des ruptures de stock de contraceptifs importantes entre 1997 et 1998. D'octobre 1998 à juillet 1999, les ruptures de stocks dans les centres de soins de santé primaire et dans les cliniques de «sagesfemmes» étaient relativement élevées (voir tableau 1). Cependant, la prévalence contraceptive et le nombre de structures de santé offrant des contraceptifs n'a pas varié de manière significative entre 1997 et 1999.

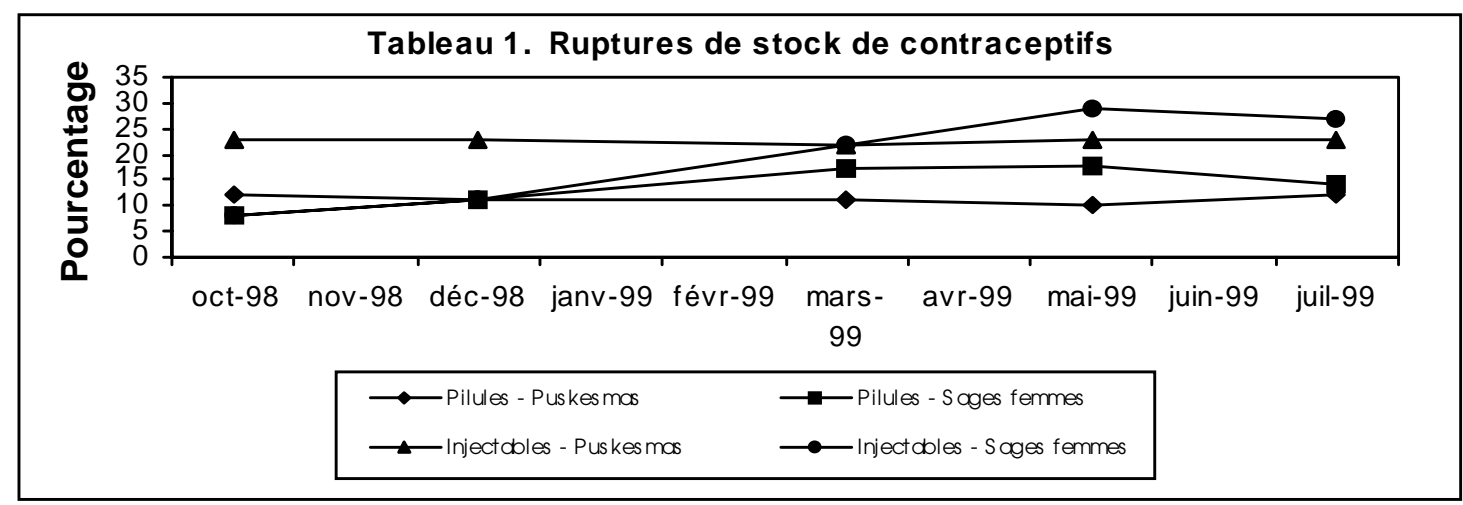


- L'incidence des infections sexuellement transmises a augmenté de 1997 à 1998, mais on ne sait pas si cette tendance est liée à la crise économique.

- Le pourcentage d'enfants des zones urbaines et rurales avec un faible poids - signe chronique de malnutrition - était plus élevé en 1998 qu'en 1995 (voir tableau 2).

Tableau 2. Pourcentage d'enfants âgés de 6 à 17 mois qui ont un poids faible pour leur âge, 1989-98.

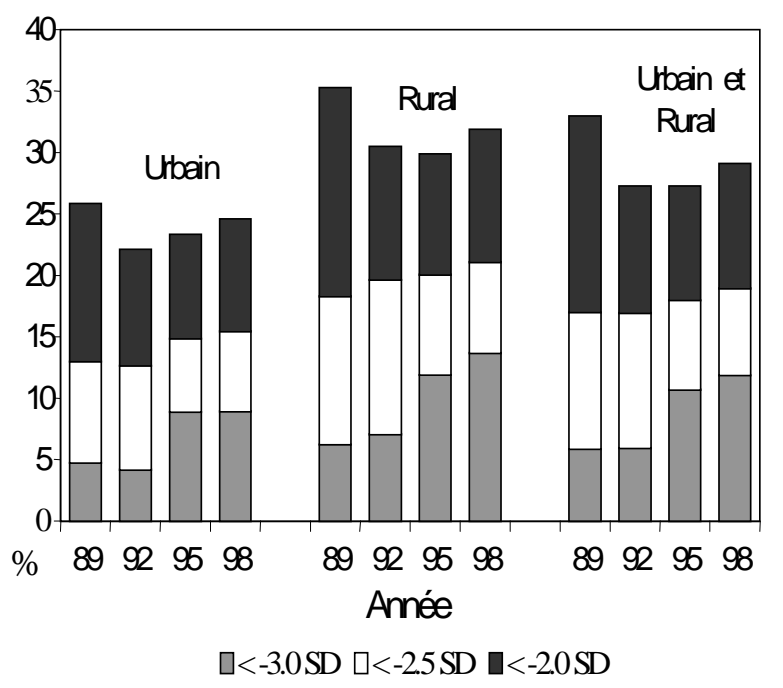

\section{Implications pour les programmes}

- Le gouvernement, les organisations non gouvernementales, les bailleurs de fonds, et les chercheurs doivent coordonner la planification des recherches importantes afin de s'assurer que les évaluations utiles et comparables.

Les responsables de programme et autres décideurs doivent tenir compte des limites de chaque banque de données avant de procéder à une généralisation sur une population plus importante ou avant d'établir une relation entre les indicateurs de santé et les tendances socioéconomiques. Ils doivent aussi comprendre que les différentes méthodes de collecte de données ont des avantages et des inconvénients.

Afin de s'assurer que les résultats de la recherche soient utiles aux responsables de programmes, les chercheurs doivent veiller à ce que les conclusions, la méthodologie de recherche, et la taille des échantillons soient indiquées de manière très précise. Il faut que les indicateurs soient comparables pour des séries chronologiques. Afin de distinguer les tendances nouvelles des effets de variations à courts termes, les chercheurs doivent analyser au moins trois données et utiliser les techniques d'analyse de tendances. Les tests de signification statistique sont indispensables à la détermination des véritables différences entre les groupes.

Gardner, Michelle et Lila Amaliah, 1999. « Analyse des résultats de recherche contradictoires sur la crise ». Pour de plus amples informations contacter : Population Council, Sanga Rchana, 53, Lodi Estate, $3^{\text {rd }}$ floor, New Delhi, 110003, India; Tel. 91-11461-10912; Fax 91-11-461-0912; E-mail: frontiers@ pcindia.org; or Population Council Indonesia, Menara Dea Building, Suite 303, Jl. Mega Kunigan Barat Dav. E4.3, No. 1, Jakarta, 12950; Tel. 6221-576-1011; Fax: 6221-576-1013; E-mail: pcjkt@cbn.net.id.

Ce projet a été réalisé avec le concours de L'AGENCE AMERICAINE POUR LE DEVELOPPEMENT INTERNATIONAL (USAID) aux termes de l'accord de coopération numéro HRN-A-00-98-00012-00.

\section{(1) Popdalibin Cound}

